\title{
Long non-coding RNA USP30-AS1 aggravates the malignant progression of cervical cancer by sequestering microRNA-299-3p and thereby overexpressing PTP4A1
}

\author{
MENGYUE CHEN $^{1}$, YUGANG CHI ${ }^{2}$, HONGWEI CHEN ${ }^{1}$ and LIMEI ZHAO ${ }^{1}$ \\ ${ }^{1}$ Department of Gynaecology, The First People's Hospital of Chongqing Liangjiang New Area, \\ Chongqing 401120; ${ }^{2}$ Department of Gynaecology and Obstetrics, Chongqing Health Center for \\ Women and Children, Chongqing 400021, P.R. China
}

Received December 19, 2020; Accepted April 8, 2021

DOI: 10.3892/ol.2021.12766

\begin{abstract}
USP30 antisense RNA 1 (USP30-AS1) has been studied in bladder urothelial carcinoma. However, the detailed role of USP30-AS1 in cervical cancer remains to be elucidated. Therefore, the present study determined whether USP30-AS1 is implicated in cervical cancer malignancy, and investigated relevant molecular mechanisms. USP30-AS1 expression was measured via reverse transcription-quantitative PCR. Functional experiments, including the Cell Counting Kit- 8 assay, flow cytometry, Transwell migration and invasion assays, and mouse tumour model, were performed in order to elucidate the roles of USP30-AS1. The target of USP30-AS1 was predicted using bioinformatics analysis, which was further verified via RNA immunoprecipitation and luciferase reporter assays. Herein, USP30-AS1 overexpression was detected in cervical cancer sample data from The Cancer Genome Atlas and our cohort. Patients with cervical cancer expressing high levels of USP30-AS1 exhibited shorter overall survival than those with low USP30-AS1 expression. In vitro and in vivo experiments revealed that USP30-AS1 interference promoted cell apoptosis; restrained cell proliferation, migration and invasion in vitro, and hindered tumour growth in vivo. Mechanistically, USP30-AS1 competed for microRNA-299-3p (miR-299-3p) in cervical cancer and lowered the regulatory actions of miR-299-3p on protein tyrosine phosphatase type IVA (PTP4A1), resulting in PTP4A1 overexpression. Furthermore, rescue experiments confirmed that miR-299-3p interventions or exogenous PTP4A1 could counteract the cancer-inhibiting actions of USP30-AS1 silencing on cervical cancer cells. In conclusion, the miR-299-3p/PTP4A1 axis is
\end{abstract}

Correspondence to: Dr Limei Zhao, Department of Gynaecology, The First People's Hospital of Chongqing Liangjiang New Area, 199 Renxing Road, Chongqing 401120, P.R. China

E-mail: gynaec_zhaolimei@126.com

Key words: non-coding RNA, competing endogenous RNA, cervical cancer, microRNAs the downstream effector of USP30-AS1 in cervical cancer, forming the USP30-AS1/miR-299-3p/PTP4A1 pathway. This newly identified competing endogenous RNA pathway may offer a novel theoretical and experimental basis for developing promising new strategies for the targeted therapy of cervical cancer.

\section{Introduction}

Cervical cancer, one of the most common gynaecological tumours, is the second-most common cancer among women and the fourth leading cause of tumour-associated deaths in females worldwide (1). Statistics indicate that over half a million new cervical cancer cases occur every year worldwide, and $85 \%$ of these occur in developing and underdeveloped countries (2). Extensive cervical cancer screening has drastically decreased the morbidity from cervical cancer in the past decade. However, cervical cancer still substantially influences women's health, and will remain an important public health issue for several decades (3). Currently, the primary treatments for cervical cancer are a radical hysterectomy with pelvic lymph node dissection, chemotherapy, and radiotherapy (4). To date, despite improvements in the clinical outcomes of cervical cancer following first-line treatments, its prognosis remains unsatisfactory (5). Furthermore, distant metastasis and tumour recurrence make cervical cancer a highly malignant and deadly human cancer (6). Therefore, investigating the molecular mechanisms underlying cervical cancer pathogenesis is urgently required in order to develop effective targeted treatments.

The expression profiles and roles of long non-coding RNAs (lncRNAs) in human cancers are a research focus in the oncology field (7). IncRNAs are a subcategory of transcripts that are $>200$ nucleotides in length (8). They have no protein-coding ability but participate in regulating gene expression at both the transcriptional and post-transcriptional levels (9). IncRNAs have been extensively studied and identified as novel modulators during carcinogenesis and cancer progression (10-12). The aberrant expression of lncRNAs has been widely discovered in human cancers, manifesting a close correlation with aggressive biological events (13-15). Several studies have demonstrated the crucial regulatory actions 
of lncRNAs in modulating pathological conditions during cervical carcinogenesis and cancer progression (16-18).

MicroRNAs (miRNAs/miRs) are another group of single-stranded RNA molecules with 17-23 nucleotides. They lack protein-coding ability (19) but directly interact with the 3'-untranslated regions (3'-UTRs) of their target genes and subsequently trigger mRNA degradation and/or translational repression (20). Recently, several miRNAs have been confirmed to be dysregulated and perform important regulatory actions towards cervical cancer oncogenesis by exerting tumour-inhibiting or tumour-facilitating roles $(21,22)$. Recently, a novel regulatory mechanism, called the competing endogenous RNA (ceRNA) theory, was uncovered, based on which IncRNAs can decoy certain miRNAs and thus indirectly affect the miRNA's target genes (23). Therefore, therapies specifically against lncRNAs or miRNAs may be promising therapeutic techniques for cervical cancer.

A novel lncRNA, called USP30 antisense RNA 1 (USP30-AS1), has been studied in bladder urothelial carcinoma (24). Utilizing The Cancer Genome Atlas (TCGA) database, it was revealed that USP30-AS1 was one of the most dysregulated lncRNAs in cervical squamous cell carcinoma and endocervical adenocarcinoma (CESC), suggesting that USP30-AS1 may exert important roles during cervical cancer progression. Additionally, the detailed role of USP30-AS1 in cervical cancer remains unknown. Therefore, the present study aimed to determine the potential functions of USP30-AS1 in cervical cancer and uncover its underlying molecular events.

\section{Materials and methods}

Human samples. Tumour tissues and matched adjacent normal tissues were obtained from 56 patients with cervical cancer (age range, 41-73 years) at The First People's Hospital of Chongqing Liangjiang New Area (Chongqing, China). Patients with International Federation of Gynaecology and Obstetrics stage (25) I-II and III-IV were 22 and 34, respectively. Immediately after tissue excision, samples were frozen in liquid nitrogen and stored in liquid nitrogen $\left(-196^{\circ} \mathrm{C}\right)$ until further use. The follow-up lasted for 60 months. Patients that had received radiochemotherapy were excluded. Patients with other types of human cancer were also excluded from the study. All experimental procedures were approved by the Human Ethics Approval Committee of The First People's Hospital of Chongqing Liangjiang New Area (approval no. ECAC-TFHCQLJ.20150601). Furthermore, written informed consent was obtained from all patients before the study.

TCGA program. TCGA dataset of CESC (TCGA-CESC) (26) was downloaded from TGCA (https://tcga-data.nci.nih. gov/tcga/), and used in the expression analysis of USP30-AS1 and miR-299-3p. The dataset included 306 CESC tissues and 3 normal cervical tissues. In addition, the clinicopathological features of these cohorts were obtained, and the correlation between USP30-AS1/miR-299-3p expression and clinicopathological features in CESC was also assessed. The survival analysis of PTP4A1 in TCGA-CESC cohorts was implemented using The Human Protein Atlas (https://www. proteinatlas.org/).
Cell culture. The normal human cervical epithelial cell line Ect1/E6E7 was acquired from the American Type Culture Collection (ATCC). It was cultured in keratinocyte serum-free medium (cat. no. 17005-042; Gibco; Thermo Fisher Scientific, Inc.) supplemented with $0.1 \mathrm{ng} / \mathrm{ml}$ human recombinant epidermal growth factor, $0.05 \mathrm{mg} / \mathrm{ml}$ bovine pituitary extract, and $0.4 \mathrm{mM}$ calcium chloride. The three human cervical cancer cell lines, SiHa (ATCC), HeLa (ATCC) and CaSki (Cell Bank of the Chinese Academy of Sciences), were cultured in RPMI-1640 medium (Gibco; Thermo Fisher Scientific, Inc.). Another cervical cancer cell line, C-33A (Cell Bank of the Chinese Academy of Sciences), was maintained in minimum essential medium (Gibco; Thermo Fisher Scientific, Inc.). All culture media were supplemented with $10 \%$ foetal bovine serum (FBS; Gibco; Thermo Fisher Scientific, Inc.) and 1\% penicillin-streptomycin (Gibco; Thermo Fisher Scientific, Inc.). Cells were cultured at $37^{\circ} \mathrm{C}$ in humidified air with $5 \% \mathrm{CO}_{2}$.

Transfection experiments. Small interfering (si)RNAs against USP30-AS1 (si-USP30-AS1s) were designed to knockdown USP30-AS1 expression using scrambled control (si-NC) as the control. All siRNAs were constructed by Shanghai GenePharma Co., Ltd. The si-USP30-AS1 sequences were as follows: si-USP30-AS1\#1, 5'-CTGCTATAATTAGTT ATTATTGT-3'; si-USP30-AS1\#2, 5'-AGCCAACAAACT TATTGTTTACT-3'; and si-USP30-AS1\#3, 5'-TTCATT ATTTACATTTAATATTC-3'. The si-NC sequence was 5'-CACGATAAGACA ATGTAT TT-3'. miRNA-299-3p (miR-299-3p) mimic, miRNA scrambled control (miR-NC), miR-299-3p inhibitor (anti-miR-299-3p), and miRNA inhibitor control (anti-miR-NC) were also synthesised by Shanghai GenePharma Co., Ltd. The miR-299-3p mimics sequence was 5'-UUCGCCAAAUGGUAGGGUGUAU-3' and the miR-NC sequence was 5'-UUGUACUACACAAAAGUACUG-3'. The anti-miR-299-3p inhibitor sequence was 5'-AAGCGGUUU ACCAUCCCACAUA-3' and the anti-miR-NC sequence was 5'-ACUACUGAGUGACAGUAGA-3'. The protein tyrosine phosphatase type IVA (PTP4A1)-overexpressing vector pcDNA3.1-PTP4A1 (pc-PTP4A1; GeneChem Co., Ltd.) was produced by cloning PTP4A1 sequences into pcDNA3.1. Cells were placed into 6-well plates and grown to 60-70\% confluency, followed by transfection with siRNA (100 pmol), miRNA oligonucleotides $(100 \mathrm{pmol})$ or plasmids $(4 \mu \mathrm{g})$ using Lipofectamine $^{\mathrm{TM}} 2000$ (Invitrogen; Thermo Fisher Scientific, Inc.). All transfections were performed at room temperature. Reverse transcription-quantitative PCR (RT-qPCR), flow cytometric analysis, and Transwell migration and invasion assays and western blotting were executed at $48 \mathrm{~h}$ post-transfection. After $24 \mathrm{~h}$ culture at $37^{\circ} \mathrm{C}$, Cell Counting kit-8 (CCK-8) assay was performed.

$R T$ - $q P C R$. Total RNA was isolated from tissues or cultured cells using TRIzol ${ }^{\circledR}$ reagent (Invitrogen; Thermo Fisher Scientific, Inc.). To quantify USP30-AS1 and PTP4A1 expression, total RNA was reverse transcribed to complementary DNA (cDNA) using the PrimeScript ${ }^{\mathrm{TM}}$ RT Reagent kit with gDNA Eraser (both from Takara Biotechnology Co., Ltd.). The thermocycling conditions were as follows: $37^{\circ} \mathrm{C}$ for $15 \mathrm{~min}$, and $85^{\circ} \mathrm{C}$ for $5 \mathrm{sec}$. PCR amplification was performed using 
TB Green ${ }^{\circledR}$ Premix Ex Taq ${ }^{\mathrm{TM}}$ II (Takara Biotechnology Co., Ltd.). The thermocycling conditions were as follows: $95^{\circ} \mathrm{C}$ for $30 \mathrm{sec}, 95^{\circ} \mathrm{C}$ for $5 \mathrm{sec}, 60^{\circ} \mathrm{C}$ for $30 \mathrm{sec}$, for 40 cycles. GAPDH functioned as an internal control for USP30-AS1 and PTP4A1. To detect miR-299-3p expression, cDNA was obtained from total RNA via RT using the miRcute miRNA First-Strand cDNA Synthesis kit (Tiangen Biotech Co., Ltd.). The thermocycling conditions were as follows: $42^{\circ} \mathrm{C}$ for $60 \mathrm{~min}$, and $95^{\circ} \mathrm{C}$ for $3 \mathrm{~min}$. The miRcute miRNA qPCR Detection kit SYBR Green (Tiangen Biotech Co., Ltd.) was used for qPCR. The thermocycling conditions were as follows: $95^{\circ} \mathrm{C}$ for $15 \mathrm{~min}$, $94^{\circ} \mathrm{C}$ for $20 \mathrm{sec}, 60^{\circ} \mathrm{C}$ for $34 \mathrm{sec}$, for 45 cycles. The miRNA expression was normalized to that of U6 small nuclear RNA. The relative gene expression was calculated using the $2^{-\Delta \Delta \mathrm{C} q}$ method (27). The following primer sequences were used: USP30-AS1 forward, 5'-GTCTCCCCAGGTCTGTGCTTA A-3' and reverse, 5'-GTATTTTTTCCTTATGCTGCCAAA-3'; PTP4A1 forward, 5'-ACCAATGCGACCTTAAACAAATT-3' and reverse, 5'-CTTCTTTCTCCACAAGAGTAGTGTCAT-3'; GAPDH forward, 5'-ACCTGACCTGCCGTCTAGAAAA-3' and reverse, 5'-TTGAAGTCAGAGGAGACCACCTG-3'; U6 forward, 5'-CTCGCTTCGGCAGCACA-3' and reverse, 5'-AACGCTTCACGAATTTGCGT-3'; miR-299-3p forward, 5'-TCGGCAGGUUCGCCAAAUG-3' and reverse, 5'-CAC TCAACTGGTGTCGTGGA-3'; miR-127-5p forward, 5'-TCG GCAGGCUGAAGCUCAG-3' and reverse, 5'-CACTCAACT GGTGTCGTGGA-3'; miR-186-3p forward, 5'-TCGGCAGGG GGUUUUUUAAGU-3' and reverse, 5'-CACTCAACTGGT GTCGTGGA-3'; miR-204-5p forward, 5'-TCGGCAGGUUCC CUUUGUCA-3' and reverse, 5'-CACTCAACTGGTGTCGTG GA-3'; miR-379-3p forward, 5'-TCGGCAGGUCAAUCACCU GG-3' and reverse, 5'-CACTCAACTGGTGTCGTGGA-3'; and miR-411-3p forward, 5'-TCGGCAGGCCAAUCACCU GG-3' and reverse, 5'-CACTCAACTGGTGTCGTGGA-3'.

Subcellular fractionation. Cervical cancer cells in the logarithmic growth phase were digested with $0.25 \%(\mathrm{w} / \mathrm{v})$ trypsin and centrifuged at $1,000 \times \mathrm{g}$ for $5 \mathrm{~min}$. The cytoplasmic and nuclear fractions were separated using the Cytoplasmic and Nuclear RNA Purification kit (Norgen Biotek Corp.). The relative abundances of USP30-AS1, GAPDH and U6 in both fractions were determined using RT-qPCR. GAPDH and U6 were used as the cytoplasmic and nuclear controls, respectively.

CCK- 8 assay. Infected cervical cancer cells were harvested and used to prepare a single-cell suspension. Each well of the 96-well plates was covered with $100 \mu \mathrm{l}$ cell suspension containing 2,000 cells. The CCK- 8 assay was performed at $0,1,2$ and 3 days after incubation at $37^{\circ} \mathrm{C}$ with $5 \% \mathrm{CO}_{2}$. A $10-\mu 1$ volume of CCK-8 reagent (Beyotime Institute of Biotechnology) was directly added to each well. After $2 \mathrm{~h}$, the optical density was measured at $450 \mathrm{~nm}$ (OD450) using a microplate spectrophotometer.

Flow cytometric analysis of cell apoptosis. Cervical cancer cells transfected with the aforementioned molecular products were cultivated at $37^{\circ} \mathrm{C}$ with $5 \% \mathrm{CO}_{2}$ for $48 \mathrm{~h}$ before cell apoptosis detection using the Annexin V-FITC Apoptosis Detection kit (Beyotime Institute of Biotechnology). Cells were digested with $0.25 \%$ (w/v) trypsin, centrifuged in
$1,000 \times \mathrm{g}$ at room temperature for $5 \mathrm{~min}$ and rinsed with ice-cold phosphate-buffered saline, followed by staining with $5 \mu \mathrm{l}$ of Annexin V-FITC and $10 \mu \mathrm{l}$ of propidium iodide. After $20 \mathrm{~min}$ of incubation at $20-25^{\circ} \mathrm{C}$ in the dark, apoptotic cells were identified using a flow cytometer (BD Biosciences).

Transwell migration and invasion assays. For the migration assay, a single-cell suspension was prepared from infected cervical cancer cells using serum-free culture medium. The apical chambers of 24-well Transwell ${ }^{\circledR}$ inserts (pore size, $8 \mu \mathrm{m}$; BD Biosciences) were filled with $200 \mu \mathrm{l}$ cell suspension containing $5 \times 10^{4}$ cells, whereas $600 \mu 1$ of $20 \%$ FBS-containing culture medium was added into the lower chamber. After $24 \mathrm{~h}$ culture at $37^{\circ} \mathrm{C}$, the inserts were immersed in $4 \%$ paraformaldehyde at room temperature for $30 \mathrm{~min}$ and washed twice with phosphate-buffered saline. Crystal violet staining was performed at room temperature for $30 \mathrm{~min}$. After extensive washing, the non-migrated cells were cleaned from the upper face of the inserts with a cotton swab, whereas migrated cells were imaged and counted in five visual fields using a light microscope (Olympus Corporation; x200 magnification). The cell invasion assay was performed in a similar manner, with the exception that the inserts were precoated with Matrigel (BD Biosciences).

Mouse tumour model. Short hairpin RNA (shRNA) sequences targeting USP30-AS1 and negative control shRNA (sh-NC) designed and synthesized by Shanghai GenePharma Co., Ltd., were inserted into a lentiviral vector. The vectors alongside psAX2 and pMD2G vectors were injected into 293T cells (Cell Bank of the Chinese Academy of Sciences). The lentiviral supernatant was collected after $48 \mathrm{~h}$ of cultivation and used to infect HeLa cells. Finally, the stably transfected cells were filtered using puromycin (Sigma-Aldrich; Merck KGaA).

A total of six female BALB/c nude mice (20 g; age, 4-6 weeks) were purchased from HFK Bioscience (http://www.hfkbio.com). The animals were housed under specific pathogen-free conditions at $25^{\circ} \mathrm{C}$ and $50 \%$ humidity, with a 10:14 light/dark cycle and ad libitum access to food and water. The flank of mice were subcutaneously injected with $2 \times 10^{6} \mathrm{HeLa}$ cells stably transfected with sh-USP30-AS1 or sh-NC. The condition of the mice was monitored once every 2 days, and the width and length of the subcutaneous xenografts were measured weekly. The recorded data were then used to determine the tumour volume using the following formula: Volume $=1 / 2 \mathrm{x}$ length $\mathrm{x}$ width $^{2}$. At 4 weeks after cell injection, all mice were euthanized by means of cervical dislocation. Solid tumours were excised and weighed. Protocols involving animals were approved by the Ethical Guidelines for Animal Care and Use Committee of The First People's Hospital of Chongqing Liangjiang New Area (Chongqing, China; approval no. EGACUC-TFHCQLJ.20181205). The humane endpoints used in the present study included: A tumour diameter $>15 \mathrm{~mm}$, tumour ulceration, abnormal feeding, weight loss or presence of cachexia.

Bioinformatics analysis. The potential miRNA targets of USP30-AS1 were predicted using miRDB (http://mirdb.org/). The binding interaction between PTP4A1 and miR-299-3p was predicted using TargetScan (http://www.targetscan. org/vert_60/) and miRDB. 
Dual-luciferase reporter assay. USP30-AS1 sequences containing the wild-type (WT) or mutant (MUT) binding sites for miR-299-3p were constructed by Shanghai GenePharma Co., Ltd., and cloned into the pmirGLO luciferase vector (Promega Corporation) to obtain WT-USP30-AS1 and MUT-USP30-AS1 reporter vectors. The recombinant vectors, WT-PTP4A1 and MUT-PTP4A1, were designed and produced using the same method. The resulting vectors together with miR-299-3p mimic or miR-NC were transfected into cervical cancer cells seeded into 6-well plates at a density of $6 \times 10^{5}$ cells/well. Transfection experiments were performed using Lipofectamine 2000. After $48 \mathrm{~h}$ of incubation, the injected cells were collected and measured using the Dual-luciferase Reporter Assay kit (Promega Corporation).

RNA immunoprecipitation (RIP). Cervical cancer cells were harvested and treated using the EZ-Magna RIP RNA-binding Protein Immunoprecipitation kit (cat. no. 03-110; EMD Millipore), which was performed according to manufacturer's protocol. Cells were lysed in RIP cell lysis buffer that came from the kit, and the supernatant was collected after centrifugation at $1,000 \mathrm{xg}$ and $4^{\circ} \mathrm{C}$ for $10 \mathrm{~min}$. The cell extract $(100 \mu \mathrm{l})$ was cultured, and magnetic beads conjugated with human anti-AGO2 antibody $(5 \mu \mathrm{l})$ or normal mouse $\operatorname{IgG}(5 \mu \mathrm{l})$ (both from cat. no. 03-110; EMD Millipore) were added. Cells were incubated for $12 \mathrm{~h}$ and kept at $4^{\circ} \mathrm{C}$. Finally, magnetic beads were collected and treated with proteinase $\mathrm{K}$ to remove proteins. The relative enrichments of USP30-AS1, miR-299-3p and PTP4A1 in immunoprecipitated RNA were quantified via RT-qPCR.

Western blotting. The total protein was extracted from cultured cells by cultivating the cultured cells with RIPA lysis buffer (Beyotime Institute of Biotechnology). An enhanced BCA Protein Assay kit (Beyotime Institute of Biotechnology) was used to measure protein concentrations. A total of $30 \mu \mathrm{g}$ protein were separated via SDS-PAGE ( $10 \%$ gel). The separated proteins were then transferred onto polyvinylidene difluoride membranes and blocked with $5 \%$ non-fat dried milk at room temperature for $2 \mathrm{~h}$. After overnight incubation at $4^{\circ} \mathrm{C}$ with primary antibodies targeting PTP4A1 (1:1,000 dilution; cat. no. ab121185) or GAPDH (1:1,000 dilution; cat. no. ab128915) (both from Abcam), horseradish peroxidase (HRP)-conjugated IgG secondary antibody (1:5,000 dilution; cat. no. ab205718; Abcam) was added, and cultivated at room temperature for $1 \mathrm{~h}$. Ultimately, target protein development was achieved using Immobilon ${ }^{\circledR}$ ECL Ultra Western HRP Substrate (EMD Millipore). The densitometry was implemented utilizing Quantity One software (4.62 version; Bio Rad Laboratories, Inc.).

Statistical analysis. Statistical analysis was performed using SPSS 19.0 software (SPSS, Inc.). All experiments were performed in triplicate and data are presented as the mean \pm standard error. Paired Student's t-test was used to compare the levels of genes (USP30-AS1, miR-299-3p and PTP4A1) between cervical cancer tissues and normal tissues, while unpaired Student's t-test was used to compare all other differences between two groups. One-way analysis of variance followed by Tukey's post hoc test was used to compare differences between multiple groups. The gene correlation analysis was performed using Pearson's correlation analysis. Survival curves were generated using the Kaplan-Meier method and compared using the log-rank test. $\mathrm{P}<0.05$ was considered to indicate a statistically significant difference.

\section{Results}

USP30-AS1 depletion inhibits cervical cancer cell proliferation, migration and invasion, and promotes cell apoptosis in vitro. First, USP30-AS1 expression in CESC was determined using TCGA database. A marked increase in USP30-AS1 expression was identified in CESC tissues when compared with normal tissues (Fig. 1A). Also, the correlation between USP30-AS1 expression and clinicopathological features in CESC was analysed employing TCGA database. The results are presented in Fig. S1A and B. Then, 56 pairs of cervical cancer tissue and adjacent normal tissues were collected to determine USP30-AS1 expression. USP30-AS1 was upregulated in cervical cancer tissues when compared with matched normal tissues (Fig. 1B). Furthermore, compared with Ect1/E6E7 cells, USP30-AS1 levels were increased in the tested cervical cancer cell lines (Fig. 1C). Based on the median value of USP30-AS1, all patients with cervical cancer were classified into two groups: USP30-AS1-high $(\mathrm{n}=28)$ and USP30-AS1-low $(\mathrm{n}=28)$ expression groups. The Kaplan-Meier method alongside the log-rank test was applied for the survival analysis. Patients with high USP30-AS1 expression levels had shorter overall survival times than those with low USP30-AS1 expression (Fig. 1D).

Among the four cervical cancer cell lines, HeLa and CaSki cells exhibited the highest USP30-AS1 expression levels, as evidenced by RT-qPCR. Therefore, these two cell lines were selected for subsequent functional experiments. To address the effects of USP30-AS1 in cervical cancer, USP30-AS1-targeting siRNAs were synthesized to knock down endogenous USP30-AS1 expression, with si-NC as the control. Fig. 1E depicts the efficiency of siRNAs in decreasing USP30-AS1 expression. There were two siRNAs, si-USP30-AS1\#1 and si-USP30-AS1\#2, used to avoid off-target effects. USP30-AS1 knockdown significantly inhibited HeLa and CaSki cell proliferation (Fig. 1F). Furthermore, a notable increase in cell apoptosis was observed in USP30-AS1-deficient HeLa and CaSki cells (Fig. 1G). Transwell migration and invasion assays revealed that the absence of USP30-AS1 hindered the migratory (Fig. 1H) and invasive (Fig. 1I) abilities of $\mathrm{HeLa}$ and CaSki cells. Taken together, these results suggest that USP30-AS1 functions as a carcinogenic lncRNA in cervical cancer.

USP30-AS1 directly binds to miR-299-3p and functions as a $m i R-299-3 p$ sponge in cervical cancer. In order to investigate the carcinogenic mechanism of USP30-AS1 in cervical cancer, the subcellular distribution of USP30-AS1 was examined via a subcellular fractionation assay. USP30-AS1 was mostly localized in the cytoplasm of HeLa and CaSki cells (Fig. 2A). Several studies have reported that cytoplasmic lncRNAs can function as molecular sponges for certain miRNAs and further regulate gene expression. By searching the miRDB, a total of 41 miRNAs (Fig. 2B) were predicted as potential downstream 
A

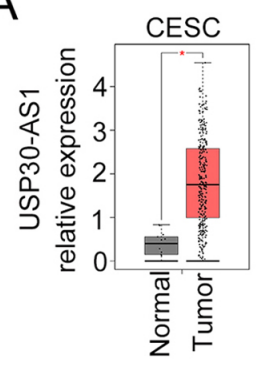

B

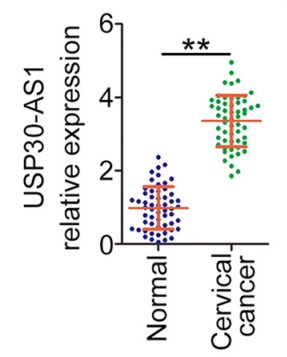

C

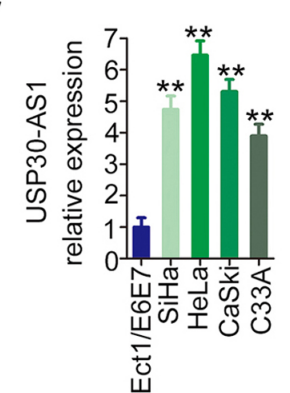

D

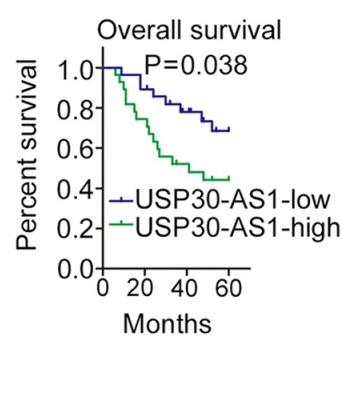

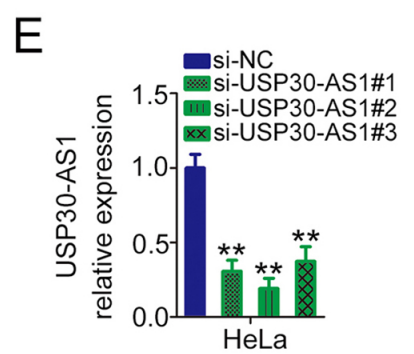

G

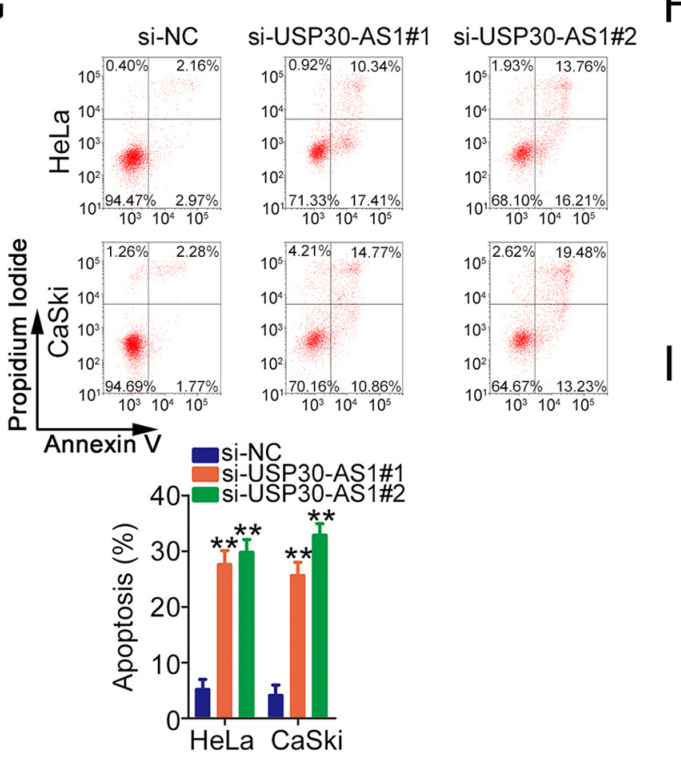

$\mathrm{F}$

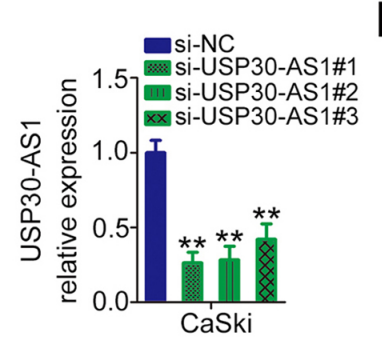

$\mathrm{H}$
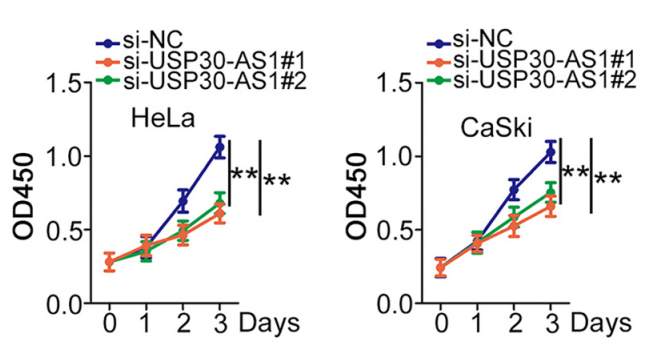
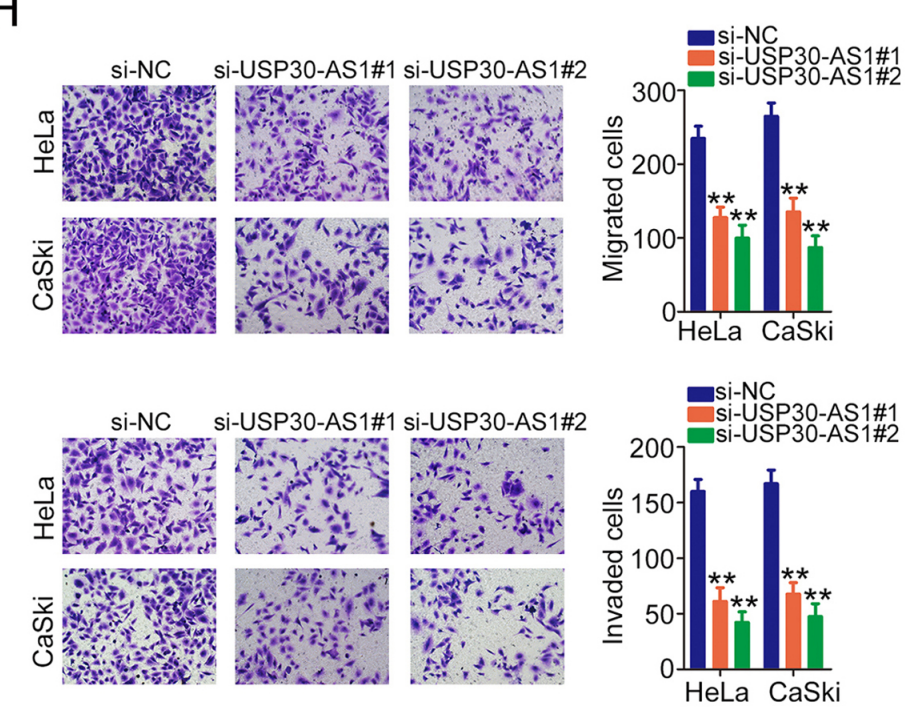

Figure 1. USP30-AS1 knockdown inhibits the malignant behaviours of cervical cancer cells. (A) USP30-AS1 expression in cervical squamous cell carcinoma and endocervical adenocarcinoma using The Cancer Genome Atlas database. ${ }^{*} \mathrm{P}<0.05$ vs. normal. (B) RT-qPCR analysis of the relative expression of USP30-AS1 in 56 cervical cancer tissues and adjacent normal tissues. ${ }^{* *} \mathrm{P}<0.01$ vs. normal. (C) USP30-AS1 expression in cervical cancer cell lines and the normal human cervical epithelial cell line Ect1/E6E7 via RT-qPCR. **P<0.01 vs. Ect1/E6E7. (D) Kaplan-Meier survival analysis showing the correlation between USP30-AS1 expression and overall survival in 56 patients with cervical cancer. (E) Transfection of si-USP30-AS1 and control si-NC was implemented in HeLa and CaSki cells. USP30-AS1 expression was detected via RT-qPCR. ${ }^{* *} \mathrm{P}<0.01$ vs. si-NC. (F and G) Proliferation and apoptosis detected using the Cell Counting Kit-8 assay and flow cytometry analysis in HeLa and CaSki cells following USP30-AS1 knockdown. ${ }^{* *} \mathrm{P}<0.01 \mathrm{vs}$. si-NC. (H and I) Transwell migration and invasion assays illustrating the migration and invasion of USP30-AS1-silenced HeLa and CaSki cells. " $\mathrm{P}<0.01$ vs. si-NC. RT-qPCR, reverse transcription-quantitative PCR; si, small interfering; NC, negative control; CESC, cervical squamous cell carcinoma and endocervical adenocarcinoma; si, small interfering.

targets of USP30-AS1. These miRNAs were analysed using TCGA database to screen miRNAs that are expressed at low levels in cervical cancer. A total of six miRNAs (miR-127-5p, miR-186-3p, miR-204-5p, miR-299-3p, miR-379-3p and miR-411-3p) were downregulated in CESC (Fig. 2C); therefore, they were selected for further investigation. Next, RT-qPCR was performed to measure the expression levels of these candidate miRNAs in cervical cancer cells following USP30-AS1 silencing. Transfection with si-USP30-AS1 resulted in markedly high expression levels of miR-299-3p in HeLa and CaSki cells (Fig. 2D). Furthermore, miR-299-3p was expressed at low levels in cervical cancer tissues (Fig. 2E) and exhibited an inverse expression correlation with USP30-AS1 (Fig. 2F). Furthermore, the correlation between miR-299-3p expression and clinicopathological features in CESC was analysed via TCGA database. The results are presented in Fig. S1C-E. Low miR-299-3p expression was significantly associated with tumour stage in patients with CESC.

Luciferase reporter and RIP assays were performed to verify the functional interaction between USP30-AS1 and miR-299-3p (Fig. 2G). The luciferase reporter assay revealed that the luciferase activity of WT-USP30-AS1 was alleviated by the miR-299-3p mimic. However, exogenous miR-299-3p expression did not affect MUT-USP30-AS1 activity (Fig. 2H). 
A

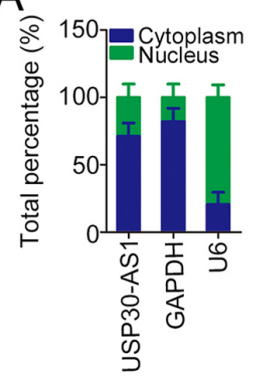

HeLa

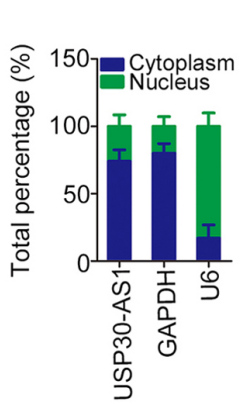

CaSki
B

\begin{tabular}{|l|l|l|l|}
\hline hsa-miR-6791-5p & hsa-miR-30a-5p & hsa-miR-5583-5p & hsa-miR-1273h-5p \\
\hline hsa-miR-4292 & hsa-miR-3960 & hsa-miR-3129-3p & hsa-miR-4684-5p \\
\hline hsa-miR-4742-3p & hsa-miR-23b-5p & hsa-miR-5584-5p & hsa-miR-299-3p \\
\hline hsa-miR-2467-3p & hsa-miR-4495 & hsa-miR-6847-5p & hsa-miR-6788-3p \\
\hline hsa-miR-6759-5p & hsa-miR-8072 & hsa-miR-4308 & hsa-miR-571 \\
\hline hsa-miR-23a-5p & hsa-miR-10400-5p & hsa-miR-6779-5p & hsa-miR-10527-5p \\
\hline hsa-miR-30e-5p & hsa-miR-7106-5p & hsa-miR-3689c & hsa-miR-5692a \\
\hline hsa-miR-30d-5p & hsa-miR-4467 & hsa-miR-3689b-3p & hsa-miR-3678-3p \\
\hline hsa-miR-30c-5p & hsa-miR-6780a-5p & hsa-miR-3689a-3p & hsa-miR-6124 \\
\hline hsa-miR-30b-5p & hsa-miR-3650 & hsa-miR-30b-3p & hsa-miR-7851-3p \\
\hline & & & hsa-miR-3190-5p \\
\hline
\end{tabular}

C

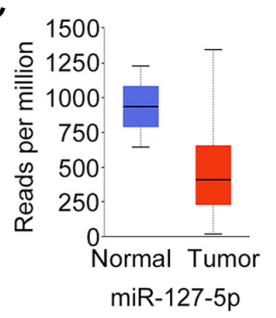

$\mathrm{D}$
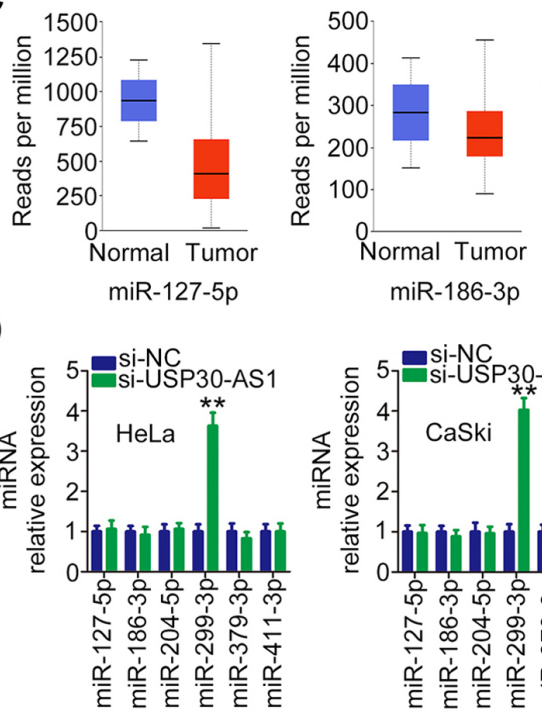

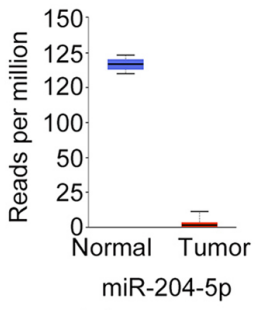

$\mathrm{E}$
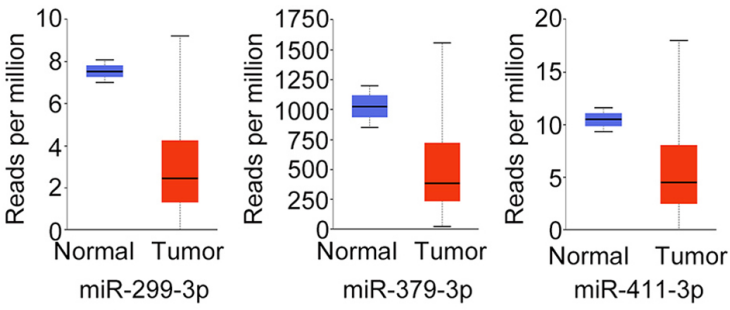

$\mathrm{F}$

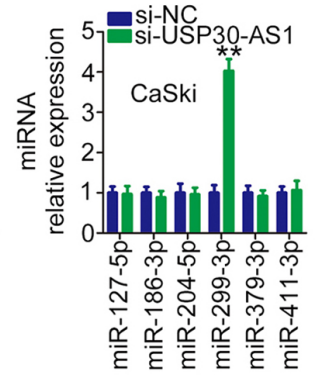

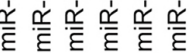
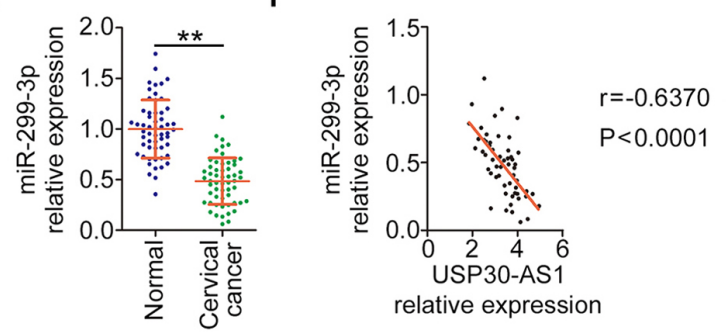

G
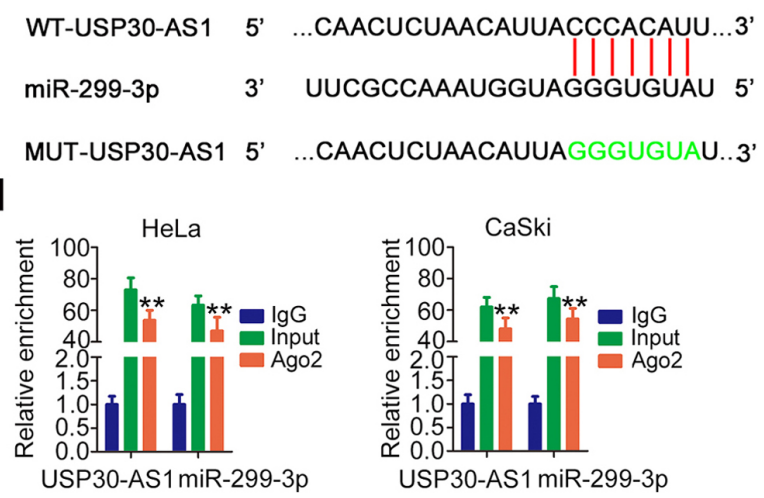

$\mathrm{H}$
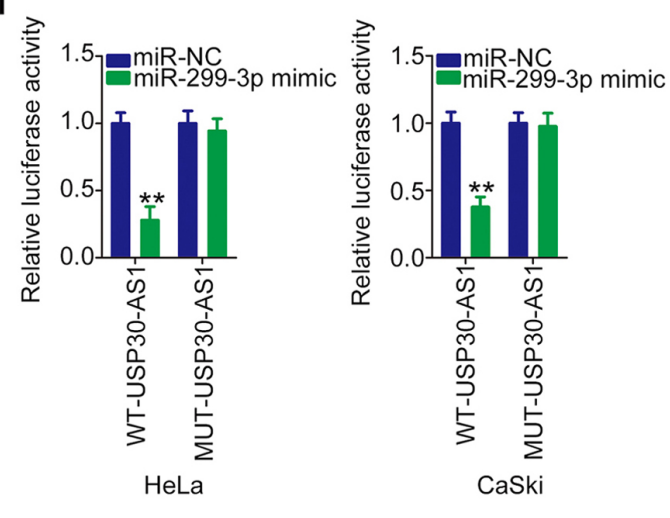

Figure 2. USP30-AS1 directly binds to miR-299-3p and sponges miR-299-3p in cervical cancer. (A) Subcellular localisation of USP30-AS1 in cervical cancer cells using the subcellular fractionation assay. (B) The target miRNAs of USP30-AS1 that were predicted by miRDB. (C) Expression analysis of the predicted miRNAs in The Cancer Genome Atlas database. A total of six miRNAs were found to be downregulated in CESC. (D) miR-299-3p expression in HeLa and CaSki cells with USP30-AS1 knockdown via RT-qPCR. ${ }^{* *} \mathrm{P}<0.01$ vs. si-NC. (E) RT-qPCR illustrating miR-299-3p expression in 56 cervical cancer tissues and adjacent normal tissues. ${ }^{* *} \mathrm{P}<0.01$ vs. normal. (F) Association between USP30-AS1 and miR-299-3p in 56 cervical cancer tissues using Pearson's correlation analysis. (G) Predicted wild-type binding sequences between USP30-AS1 and miR-299-3p and the mutant site. (H) Luciferase reporter assay to verify the binding interaction of USP30-AS1 to miR-299-3p. The luciferase activity was measured in HeLa and CaSki cells cotransfected with miR-299-3p mimic or miR-NC and WT-USP30-AS1 or MUT-USP30-AS1. * $\mathrm{P}<0.01$ vs. miR-NC. (I) RIP assay implemented to evaluate the relative enrichment of USP30-AS1 and miR-299-3p by the anti-Ago2 antibody in HeLa and CaSki cells. ${ }^{* *} \mathrm{P}<0.01$ vs. IgG. miRNA, microRNA; CESC, cervical squamous cell carcinoma and endocervical adenocarcinoma; RT-qPCR, reverse transcription-quantitative PCR; si, small interfering.

Furthermore, the RIP assay revealed that both USP30-AS1 and miR-299-3p had a tendency to be present in Ago2-rich magnetic beads (Fig. 2I). Taken together, the results suggest that USP30-AS1 is an endogenous miR-299-3p sponge in cervical cancer.
miR-299-3p plays an anti-oncogenic role and directly targets PTP4A1 in cervical cancer. In order to detect the role of miR-299-3p in cervical cancer, miR-299-3p was overexpressed in HeLa and CaSki cells following transfection with miR-299-3p mimic (Fig. 3A). Overexpressed miR-299-3p evidently 
A

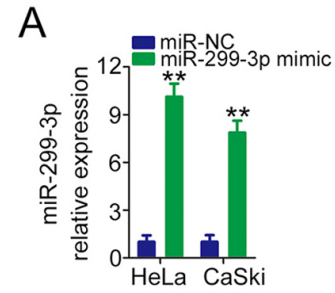

D
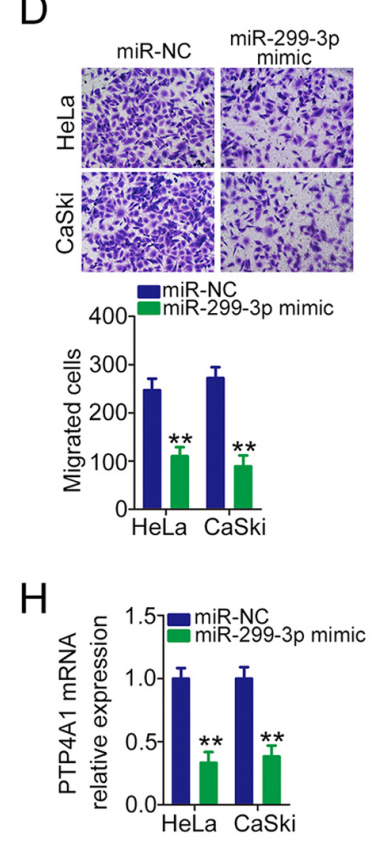

B

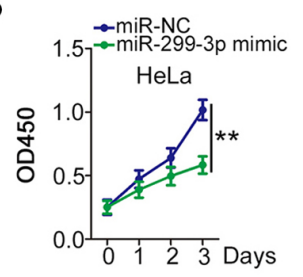

E

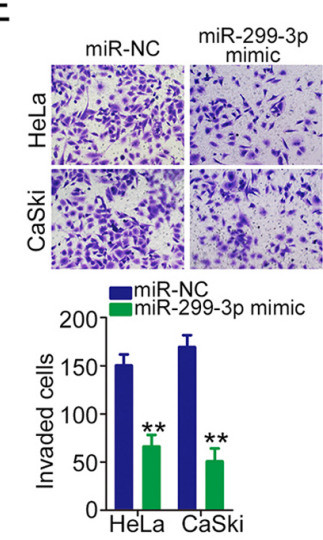

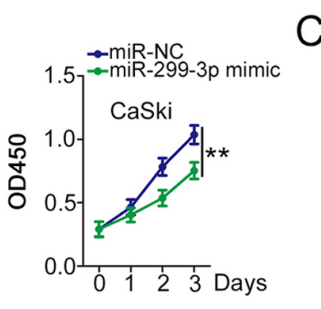

F miR-299-3p 3' UUCGCCAAAUgGUAGGGUGUAU 5,

G MUT-PTP4A1 $5{ }^{\prime}$....UUUAUGUUUACAUCUGgGUGUAU...3'

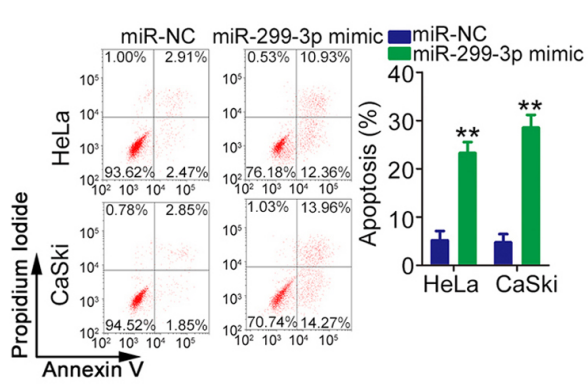

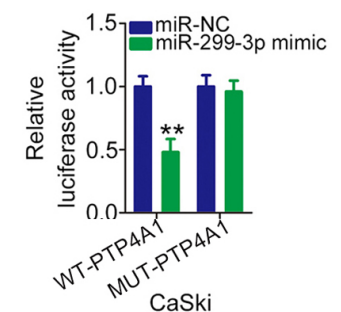

I

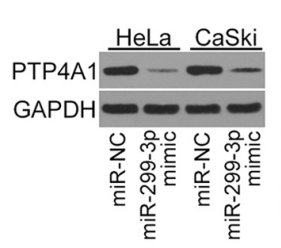

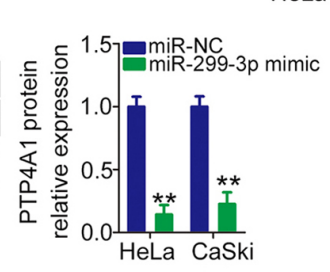

$\mathrm{J}$

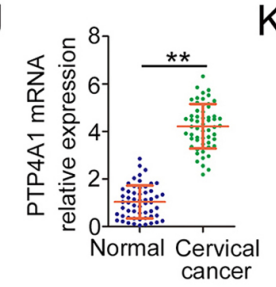

$\mathrm{K}$

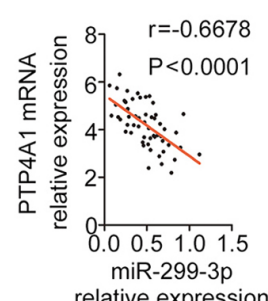

Figure 3. PTP4A1 is a direct target of miR-299-3p in cervical cancer. (A) miR-299-3p mimic or miR-NC was transfected into HeLa and CaSki cells, and miR-299-3p expression analysis was performed using RT-qPCR. ${ }^{* *} \mathrm{P}<0.01$ vs. miR-NC. (B and C) Effects of miR-299-3p upregulation on proliferation and apoptosis using the Cell Counting Kit- 8 assay and flow cytometry analysis, respectively. ${ }^{* *} \mathrm{P}<0.01$ vs. miR-NC. (D and E) Transwell migration and invasion assays to determine the migration and invasion of HeLa and CaSki cells following miR-299-3p mimic transfection. ${ }^{* *} \mathrm{P}<0.01 \mathrm{vs}$. miR-NC. (F) Predicted binding sequences of miR-299-3p to the 3'-untranslated region of PTP4A1 and mutated binding sequences. (G) Luciferase activity determined in HeLa and CaSki cells after cotransfection with miR-299-3p mimic or miR-NC and luciferase reporter vectors carrying wild-type or mutant miR-299-3p binding sites. ${ }^{* *} \mathrm{P}<0.01$ vs. miR-NC. (H and I) Regulatory actions of the miR-299-3p mimic on endogenous PTP4A1 mRNA and protein levels in HeLa and CaSki cells using RT-qPCR and western blotting, respectively. ${ }^{* *} \mathrm{P}<0.01$ vs. miR-NC. (J) PTP4A1 mRNA expression detection in 56 cervical cancer tissues and adjacent normal tissues using RT-qPCR. ${ }^{* *} \mathrm{P}<0.01$ vs. normal. (K) Pearson's correlation analysis to explore the expression correlation between PTP4A1 and miR-299-3p in 56 cervical cancer tissues. miR, microRNA; RT-qPCR, reverse transcription-quantitative PCR; NC, negative control; WT, wild-type; MUT, mutant.

lowered the proliferation ability (Fig. 3B) and facilitated the apoptosis (Fig. 3C) of HeLa and CaSki cells. Furthermore, the migration (Fig. 3D) and invasion (Fig. 3E) of HeLa and CaSki cells were obviously impaired after miR-299-3p mimic injection. Bioinformatics prediction indicated a targeting relationship between miR-299-3p and PTP4A1 (Fig. 3F). Based on the binding sequences, WT and MUT luciferase reporter vectors were designed, synthesized and cotransfected with miR-299-3p mimic or miR-NC into HeLa and CaSki cells. The reinforced expression of miR-299-3p considerably lowered WT-PTP4A1 activity, whereas miR-299-3p mimic transfection did not inhibit the luciferase activity of PTP4A1 when the binding sequences were mutated (Fig. 3G). Furthermore, PTP4A1 mRNA (Fig. 3H) and protein (Fig. 3I) expression levels were downregulated by miR-299-3p upregulation in HeLa and CaSki cells. In addition, PTP4A1 overexpression in cervical cancer tissues (Fig. 3J) was negatively correlated with miR-299-3p expression (Fig. 3K). Through TCGA database, it was revealed that high expression of PTP4A1 shows worse prognosis in cervical cancer in TCGA database (Fig. S1F). In general, miR-299-3p directly targets PTP4A1 and suppresses malignant behaviour in cervical cancer.

USP30-AS1 competitively sponges miR-299-3p to positively regulate PTP4A1 expression in cervical cancer. The present study attempted to further understand the relationship among USP30-AS1, miR-299-3p and PTP4A1 in cervical cancer. HeLa and CaSki cells were transfected with si-USP30-AS1 alone or cotransfected with anti-miR-299-3p or anti-miR-NC. PTP4A1 mRNA and protein levels were assayed by performing RT-qPCR and western blotting, respectively. USP30-AS1 interference markedly decreased PTP4A1 expression at both the mRNA (Fig. 4A) or protein (Fig. 4B) levels, whereas anti-miR-299-3p cotransfection reversed its regulatory actions on PTP4A1 expression (Fig. 4C and D). Furthermore, a RIP assay was performed to assess the potentially endogenous interaction among USP30-AS1, miR-299-3p and PTP4A1. The results revealed that all three molecules were substantially enriched by the anti-Ago2 antibody (Fig. 4E). Furthermore, a positive correlation was observed between USP30-AS1 and 
A

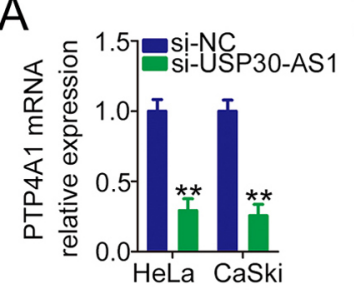

B

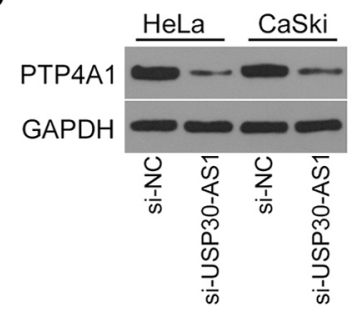

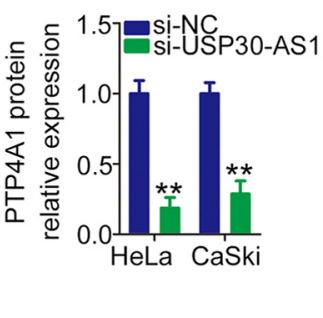

C

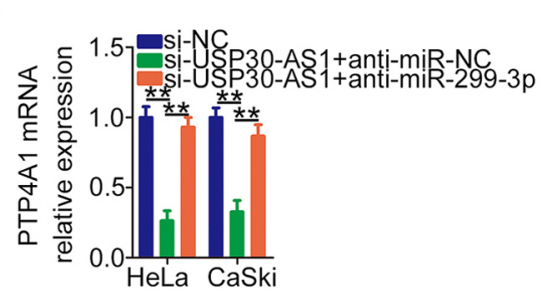

$\mathrm{D}$

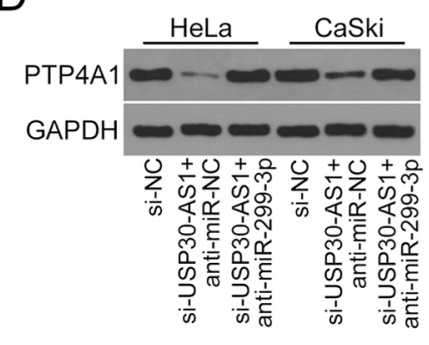

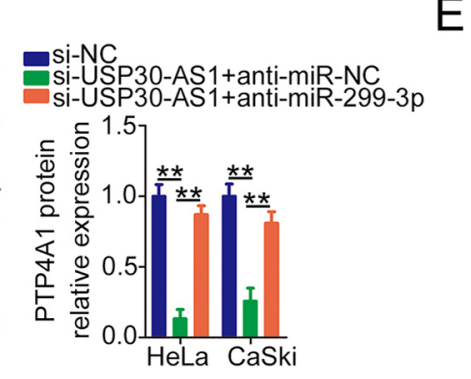

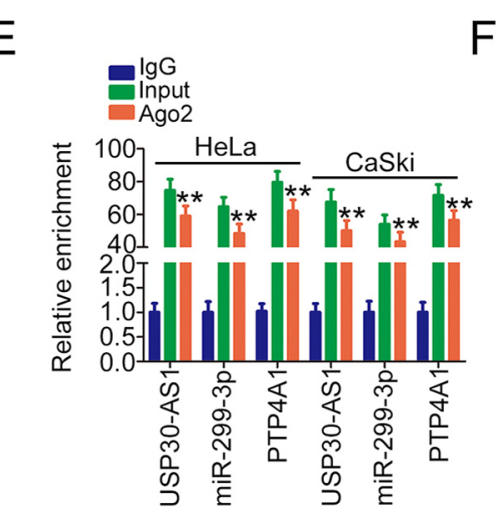

$\mathrm{F}$

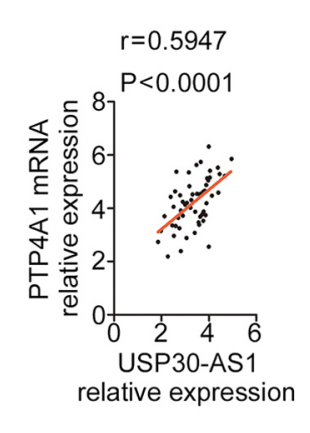

Figure 4. USP30-AS1 sequesters miR-299-3p and upregulates PTP4A1 expression in cervical cancer cells. (A and B) Reverse transcription-quantitative PCR and western blotting to detect PTP4A1 mRNA and protein levels, respectively, in HeLa and CaSki cells after USP30-AS1 silencing. ${ }^{* * *} \mathrm{P}<0.01$ vs. si-NC. (C and D) USP30-AS1-silenced HeLa and CaSki cells transfected with anti-miR-299-3p or anti-miR-NC, followed by PTP4A1 mRNA and protein level measurements. ${ }^{* *} \mathrm{P}<0.01$ vs. si-NC and si-USP30-AS1+ anti-miR-299-3p. (E) RIP assay to determine the relative enrichment of USP30-AS1, miR-299-3p and PTP4A1 by anti-Ago2 antibody in HeLa and CaSki cells. ${ }^{* *} \mathrm{P}<0.01$ vs. IgG. (F) Pearson's correlation analysis to assess the correlation between PTP4A1 and PTP4A1 levels in 56 cervical cancer tissues. miR, microRNA; NC, negative control; si, small interfering.

PTP4A1 expression in cervical cancer tissues (Fig. 4F). In short, USP30-AS1 functions as a ceRNA for miR-299-3p and thereby upregulates PTP4A1 in cervical cancer.

Increased output of miR-299-3p/PTP4A1 neutralises the actions of si-USP30-AS1 on cervical cancer cells. As the present study demonstrated that USP30-AS1 functions as a ceRNA to positively regulate PTP4A1 expression by sequestering miR-299-3p in cervical cancer, rescue experiments were performed to understand whether the tumour-promoting roles of USP30-AS1 were mediated by the miR-299-3p/PTP4A1 axis. Initially, RT-qPCR verified the efficiency of anti-miR-299-3p in decreasing miR-299-3p expression (Fig. 5A). HeLa and CaSki cells were cotransfected with si-USP30-AS1 alongside anti-miR-299-3p or anti-miR-NC, followed by functional experiments. miR-299-3p downregulation abrogated the antiproliferative (Fig. 5B) and proapoptotic (Fig. 5C) effects of si-USP30-AS1 in HeLa and CaSki cells. Similarly, anti-miR-299-3p markedly rescued the migration (Fig. 5D) and invasion (Fig. 5E) abilities of HeLa and CaSki cells hindered by USP30-AS1 deficiency.

Western blotting revealed that transfection of pc-PTP4A1 induced PTP4A1 overexpression in HeLa and CaSki cells (Fig. 6A). pc-PTP4A1 or pcDNA3.1 along with si-USP30-AS1 was transfected into HeLa and CaSki cells. In HeLa and CaSki cells, USP30-AS1 loss suppressed cell proliferation (Fig. 6B) and increased cell apoptosis (Fig. 6C), whereas PTP4A1 overexpression abolished these effects. Furthermore, cotransfection with pc-PTP4A1 reversed the inhibition of the migration (Fig. 6D) and invasion (Fig. 6E) of HeLa and CaSki cells by
si-USP30-AS1. Taken together, these results suggest that the ceRNA mechanism-based USP30-AS1/miR-299-3p/PTP4A1 network can promote cervical cancer cell malignant potential.

Depletion of USP30-AS1 restrains tumour growth in vivo. To analyse the effects of USP30-AS1 on tumour growth in vivo, HeLa cells stably expressing sh-USP30-AS1 were constructed and subcutaneously injected into nude mice to construct a mouse tumour model. The volume (Fig. 7A and B) and weight (Fig. 7C) of the subcutaneous tumours in the sh-USP30-AS1 group were evidently smaller than those in the sh-NC group. Additionally, the expression analyses revealed that USP30-AS1 was downregulated (Fig. 7D) but miR-299-3p was upregulated (Fig. 7E) in the tumours in the USP30-AS1-silenced group. Furthermore, the tumours in the sh-USP30-AS1 group had decreased PTP4A1 protein expression compared with the sh-NC group (Fig. 7F). Therefore, USP30-AS1 interference decreases the tumour growth of cervical cancer cells in vivo.

\section{Discussion}

Recently, a substantial amount of research has investigated the roles of lncRNAs in cervical cancer, and satisfactory results have been obtained (28-30). Several lncRNAs are differentially expressed in cervical cancer, which affect several aggressive properties of the tumour (31). Considering the importance of IncRNAs, they have huge potential for exploitation as targets for the diagnosis, prognosis and treatment of cancer. The ENCODE database reported that the human genome possesses 33,829 IncRNAs (32); however, the regulatory activities of 
A

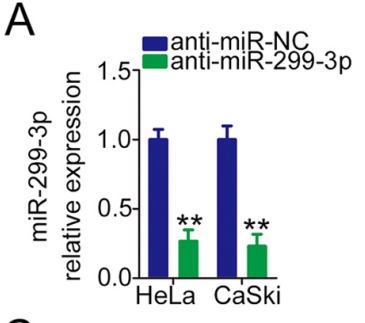

C

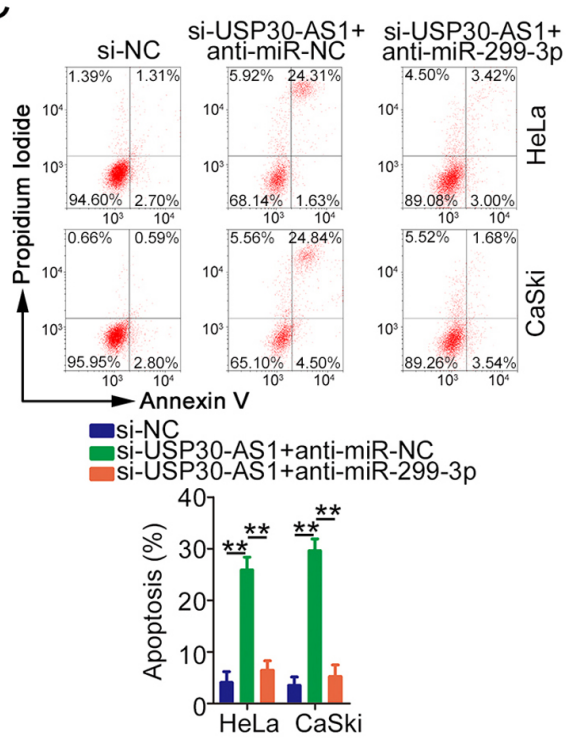

B

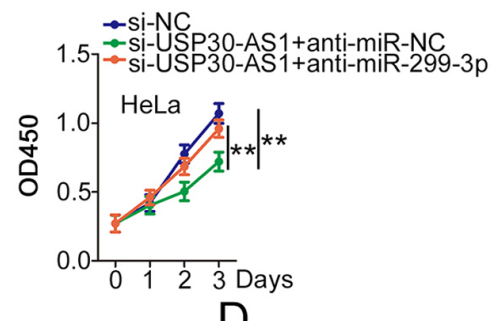

D

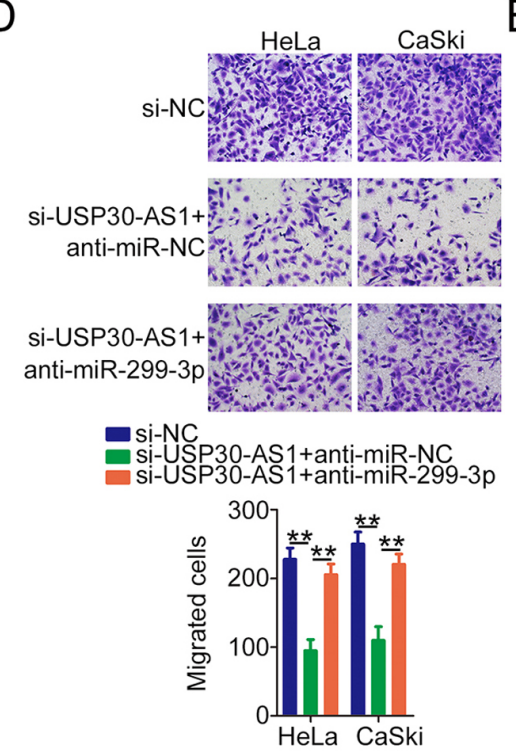

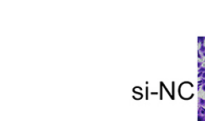

HeLa CaSki
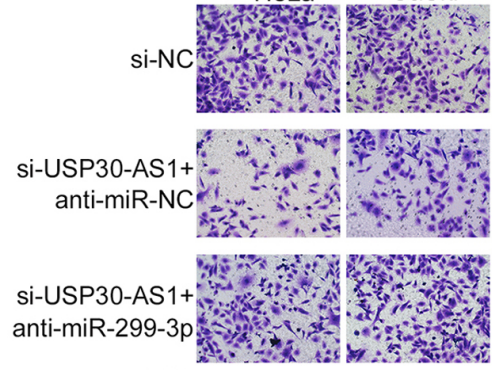

$\square$ si-NC Si-UŠ̉ $30-A S 1+$ anti-miR-NC Si-USP30-AS1+anti-miR-299-3p

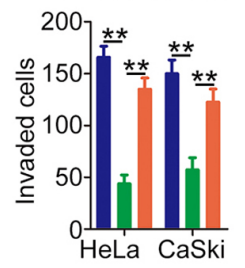

Figure 5. Regulatory actions of si-USP30-AS1 on cervical cancer cells require miR-299-3p. (A) Total RNA was isolated from anti-miR-299-3p-transfected or anti-miR-NC-transfected HeLa and CaSki cells, and miR-299-3p levels were detected via reverse transcription-quantitative PCR. ${ }^{* *} \mathrm{P}<0.01$ vs. anti-miR-NC. (B and C) USP30-AS1-silenced HeLa and CaSki cells were transfected with anti-miR-299-3p or anti-miR-NC, followed by cell proliferation and apoptosis detection using the Cell Counting Kit-8 assay and flow cytometry. ${ }^{* *} \mathrm{P}<0.01$ vs. si-NC and si-USP30-AS1+ anti-miR-299-3p. (D and E) Transwell migration and invasion assays demonstrating the migration and invasion of HeLa and CaSki cells after cotransfection with si-USP30-AS1 and anti-miR-299-3p or anti-miR-NC. ${ }^{* *} \mathrm{P}<0.01$ vs. si-NC and si-USP30-AS1+ anti-miR-299-3p. miR, microRNA; NC, negative control; si, small interfering.

most lncRNAs remain unknown, including USP30-AS1. Therefore, the present study determined whether USP30-AS1 is implicated in cervical cancer malignancy and investigated its relevant underlying molecular mechanisms.

USP30-AS1 is upregulated in bladder urothelial carcinoma and is associated with cell autophagy (24). Furthermore, lncRNAs have been verified as independent prognostic factors for bladder urothelial carcinoma (24). To date, neither the expression status nor the detailed role of USP30-AS1 have been elucidated in cervical cancer. In the present study, increased USP30-AS1 expression was observed in both cervical cancer tissues and cell lines. Furthermore, patients with cervical cancer that exhibit high USP30-AS1 expression levels had shorter overall survival than those with low USP30-AS1 expression levels. In vitro and in vivo experiments revealed that USP30-AS1 downregulation induced cell apoptosis; suppressed cell proliferation, migration and invasion in vitro; and impeded tumour growth in vivo. All these results offer a new theoretical and experimental basis for developing USP30-AS1 as a diagnostic and prognostic biomarker as well as a therapeutic target for cervical cancer.

In terms of the working mechanism, IncRNAs can compete for miRNAs through the same miRNA recognition element, which lowers the negative regulatory actions of miRNAs on target genes and indirectly modulates target mRNA levels (33). To investigate whether USP30-AS1 acts as a miRNA sponge, the exact distribution of USP30-AS1 in cervical cancer cells was determined via a subcellular fractionation assay. USP30-AS1 was identified as a cytoplasmic lncRNA in cervical cancer cells. Then, using bioinformatics analyses, the binding site between USP30-AS1 and miR-299-3p was determined, and subsequent mechanistic studies corroborated that USP30-AS1 functions as an endogenous sponge for miR-299-3p in cervical cancer. Furthermore, PTP4A1 was validated as the downstream target of miR-299-3p, and miR-299-3p could lower PTP4A1 expression in cervical cancer cells by base pairing with its 3'-UTR. Notably, further investigation determined the effects of USP30-AS1 on PTP4A1 expression in cervical cancer cells. The results of the present study suggest that PTP4A1 is indirectly regulated by USP30-AS1 by decoying miR-299-3p. Taken together, these observations unveil a new ceRNA network involving USP30-AS1, miR-299-3p and PTP4A1.

miR-299-3p dysregulation has been reported in several types of human cancer (34-36). In cervical cancer, miR-299-3p is weakly expressed and plays an anti-oncogenic role during cancer progression by inhibiting cell proliferation, colony formation and invasion (37), which is in accordance with the results of the present study. Mechanistically, PTP4A1, a prenylated protein tyrosine phosphatase, was identified as the direct target of miR-299-3p in cervical cancer. PTP4A1, also known as a phosphatase of the regenerating liver, is 
A

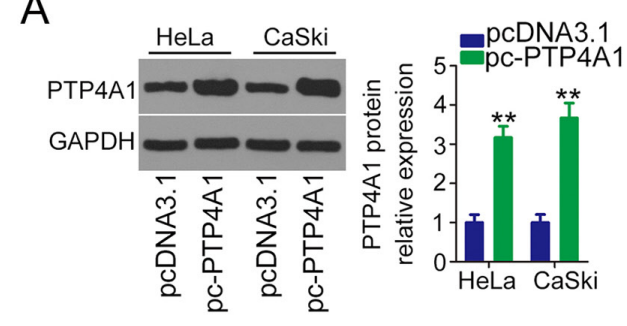

C

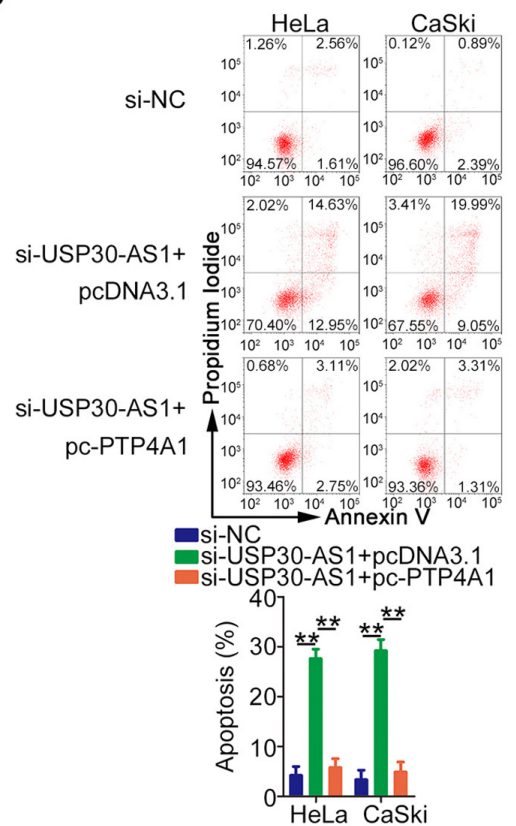

$\mathrm{B}$

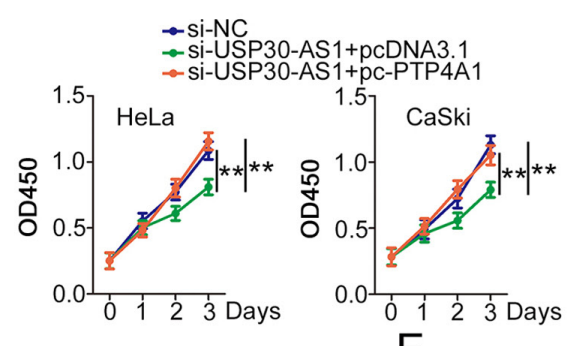

D

$\mathrm{E}$
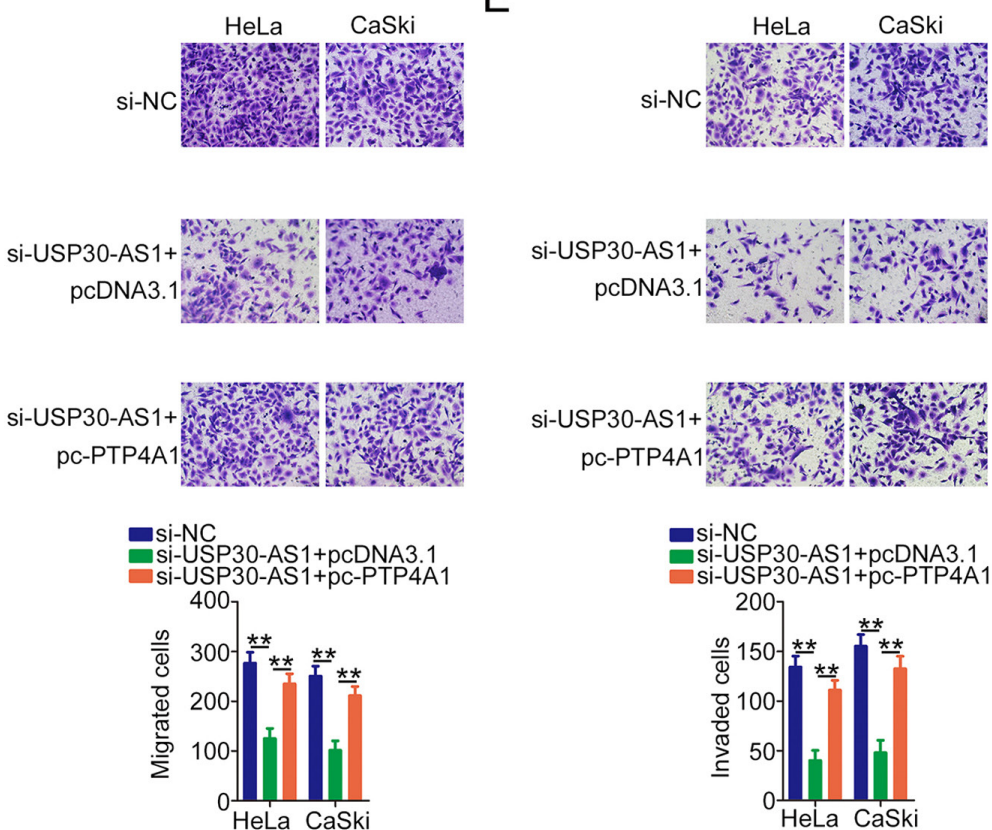

Figure 6. Overexpressing PTP4A1 reverses the antitumour activities of USP30-AS1 knockdown in cervical cancer cells. (A) Western blotting demonstrated the overexpression efficiency of pc-PTP4A1 in HeLa and CaSki cells. ${ }^{* *} \mathrm{P}<0.01$ vs. pcDNA3.1. (B-E) HeLa and CaSki cells underwent pc-PTP4A1 or pcDNA3.1 and si-USP30-AS1 cotransfection. The proliferation, apoptosis, migration and invasion of cotransfected cells were assessed using the Cell Counting Kit- 8 assay, flow cytometry, and Transwell migration and invasion assays, respectively. ${ }^{* *} \mathrm{P}<0.01$ vs. si-NC and si-USP30-AS1+ pc-PTP4A1. si, small interfering.

A

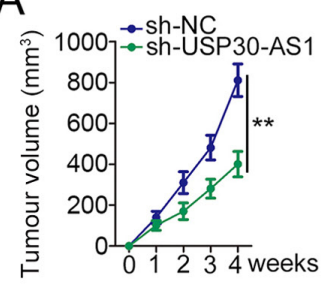

$\mathrm{E}$

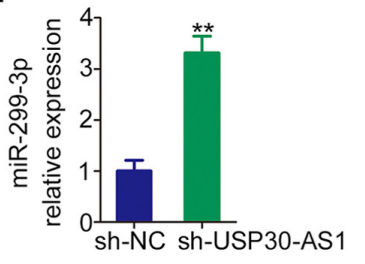

B

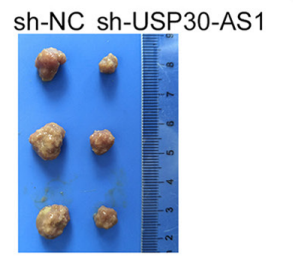

F

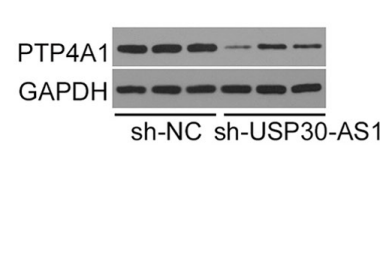

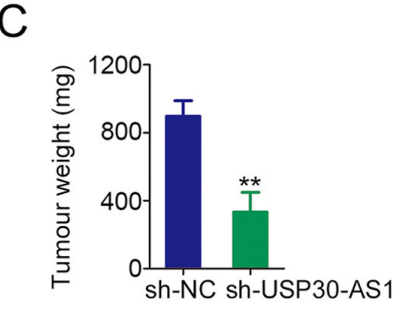
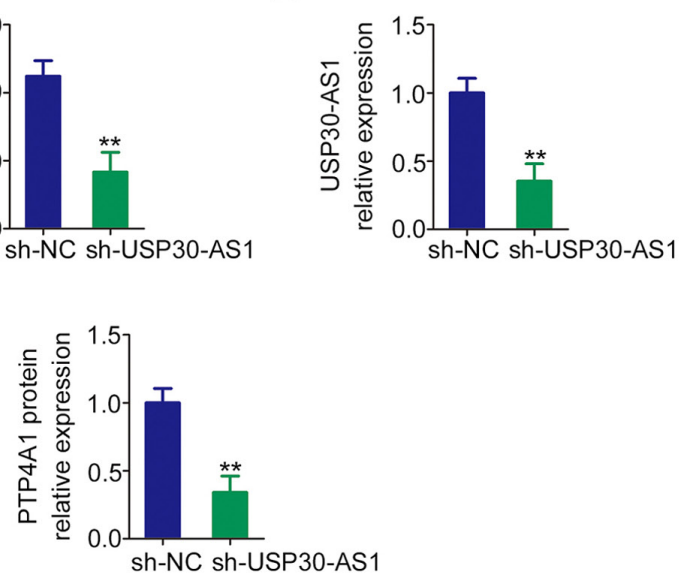

Figure 7. Absence of USP30-AS1 hinders tumour growth in vivo. (A) HeLa cells stably silenced with USP30-AS1 were subcutaneously injected into nude mice, and the growth curves were monitored based on the tumour volume detected at different time points. (B) Representative images of subcutaneous tumours collected from the sh-USP30-AS1 and sh-NC groups. (C) After nude mice were euthanized, subcutaneous tumours were excised and weighed. (D and E) USP30-AS1 and miR-299-3p expression in subcutaneous tumours obtained from the sh-USP30-AS1 and sh-NC groups was measured using reverse transcription-quantitative PCR. (F) Western blotting was used to detect PTP4A1 expression in the subcutaneous tumours. ${ }^{* *} \mathrm{P}<0.01 \mathrm{vs}$. sh-NC group. sh, short hairpin; NC, negative control; miR, microRNA.

upregulated in patients with cervical cancer and is implicated in the control of multiple malignant behaviours $(38,39)$.
Post-transcriptional regulation of PTP4A1 by miRNAs has been investigated in several types of human cancer. For 
example, miR-1271 directly targets PTP4A1 and decreases its expression in hepatocellular carcinoma, subsequently resulting in the inhibition of tumour metastasis and the epithelial-to-mesenchymal transition (40). Nevertheless, the lncRNA-mediated modulation of PTP4A1 is still far from being fully addressed in cervical cancer. To the best of our knowledge, the presented reported for the first time that PTP4A1 could be regulated by USP30-AS1 at the post-transcriptional level in cervical cancer. USP30-AS1, a type of ceRNA, competes for miR-299-3p in cervical cancer and thereby reverses the suppressive effects of miR-299-3p on PTP4A1, resulting in PTP4A1 overexpression. In addition, rescue experiments confirmed that miR-299-3p interventions or exogenous PTP4A1 counteracted the cancer-inhibiting actions of USP30-AS1 deficiency in cervical cancer cells. Taken together, the miR-299-3p/PTP4A1 axis mediates the central roles of USP30-AS1 in cervical cancer and forms the USP30-AS1/miR-299-3p/PTP4A1 pathway.

The present study had some limitations; it lacked the recurrence monitoring of all patients that participated in this research. This will be the focus of future studies.

In conclusion, to the best of our knowledge, the present study demonstrated for the first time that high USP30-AS1 expression is closely associated with worse overall survival outcomes in patients with cervical cancer. USP30-AS1 functions as a ceRNA and upregulates PTP4A1 by decoying miR-299-3p, thereby aggravating the oncogenic potential of cervical cancer cells both in vitro and in vivo. The USP30-AS1/miR-299-3p/PTP4A1 network may be an important molecular marker for cervical cancer progression and may be researched as a new strategy for targeted therapy.

\section{Acknowledgements}

Not applicable.

\section{Funding}

No funding was received.

\section{Availability of data and materials}

The datasets used and/or analysed during the present study are available from the corresponding author on reasonable request.

\section{Authors' contributions}

MC and LZ designed the present study. MC, YC, HC and LZ performed the experiments. $\mathrm{MC}$ and $\mathrm{LZ}$ confirm the authenticity of all the raw data. $\mathrm{LZ}$ analyzed the data. MC drafted the initial manuscript and revised the manuscript for important intellectual content. All authors have read and approved the final manuscript.

\section{Ethics approval and consent to participate}

All experimental procedures were approved by the Human Ethics Approval Committee of The First People's Hospital of Chongqing Liangjiang New Area (approval no. ECAC-TFHCQLJ.20150601). Furthermore, written informed consent was obtained from all patients before the study. Protocols involving animals were approved by the Ethical Guidelines for Animal Care and Use Committee of The First People's Hospital of Chongqing Liangjiang New Area (approval no. EGACUC-TFHCQLJ.20181205).

\section{Patient consent for publication}

Not applicable.

\section{Competing interests}

The authors declare that they have no competing interests.

\section{References}

1. Torre LA, Bray F, Siegel RL, Ferlay J, Lortet-Tieulent J and Jemal A: Global cancer statistics, 2012. CA Cancer J Clin 65: 87-108, 2015

2. Abbas KM, van Zandvoort K, Brisson M and Jit M: Effects of updated demography, disability weights, and cervical cancer burden on estimates of human papillomavirus vaccination impact at the global, regional, and national levels: A PRIME modelling study. Lancet Glob Health 8: e536-e544, 2020.

3. Tsikouras P, Zervoudis S, Manav B, Tomara E, Iatrakis G, Romanidis C, Bothou A and Galazios G: Cervical cancer: Screening, diagnosis and staging. J BUON 21: 320-325, 2016.

4. Nuchpramool P and Hanprasertpong J: Preoperative neutrophil-lymphocyte ratio and platelet-lymphocyte ratio are not clinically useful in predicting prognosis in early stage cervical cancer. Surg Res Pract 2018: 9162921, 2018.

5. Venkatas J and Singh M: Cervical cancer: A meta-analysis, therapy and future of nanomedicine. Ecancermedicalscience 14: 1111,2020

6. Small W Jr, Bacon MA, Bajaj A, Chuang LT, Fisher BJ, Harkenrider MM, Jhingran A, Kitchener HC, Mileshkin LR, Viswanathan AN and Gaffney DK: Cervical cancer: A global health crisis. Cancer 123: 2404-2412, 2017.

7. Bhan A, Soleimani M and Mandal SS: Long noncoding RNA and cancer: A new paradigm. Cancer Res 77: 3965-3981, 2017.

8. Mercer TR, Dinger ME and Mattick JS: Long non-coding RNAs: Insights into functions. Nat Rev Genet 10: 155-159, 2009.

9. Kornienko AE, Guenzl PM, Barlow DP and Pauler FM: Gene regulation by the act of long non-coding RNA transcription. BMC Biol 11: 59, 2013.

10. DiStefano JK: The emerging role of long noncoding RNAs in human disease. Methods Mol Biol 1706: 91-110, 2018.

11. Wapinski $\mathrm{O}$ and Chang HY: Long noncoding RNAs and human disease. Trends Cell Biol 21: 354-361, 2011.

12. Cabili MN, Trapnell C, Goff L, Koziol M, Tazon-Vega B, Regev A and Rinn JL: Integrative annotation of human large intergenic noncoding RNAs reveals global properties and specific subclasses. Genes Dev 25: 1915-1927, 2011.

13. Zhang H, Wang Y, Liu X and Li Y: Progress of long noncoding RNAs in anti-tumor resistance. Pathol Res Pract 216: 153215, 2020.

14. Teppan J, Barth DA, Prinz F, Jonas K, Pichler M and Klec C: Involvement of long non-coding RNAs (lncRNAs) in tumor angiogenesis. Noncoding RNA 6: 42, 2020.

15. Ye M,Zhang J, Wei M, Liu B and Dong K: Emerging role of long noncoding RNA-encoded micropeptides in cancer. Cancer Cell Int 20: 506, 2020.

16. Shi D, Zhang C and Liu X: Long noncoding RNAs in cervical cancer. J Cancer Res Ther 14: 745-753, 2018.

17. Aalijahan $\mathrm{H}$ and Ghorbian S: Long non-coding RNAs and cervical cancer. Exp Mol Pathol 106: 7-16, 2019.

18. Sun W, Shen NM and Fu SL: Involvement of IncRNA-mediated signaling pathway in the development of cervical cancer. Eur Rev Med Pharmacol Sci 23: 3672-3687, 2019.

19. Esquela-Kerscher A and Slack FJ: Oncomirs-microRNAs with a role in cancer. Nat Rev Cancer 6: 259-269, 2006.

20. Farazi TA, Hoell JI, Morozov P and Tuschl T: MicroRNAs in human cancer. Adv Exp Med Biol 774: 1-20, 2013. 
21. Shen S, Zhang S, Liu P, Wang J and Du H: Potential role of microRNAs in the treatment and diagnosis of cervical cancer. Cancer Genet 248-249: 25-30, 2020.

22. Miao J, Regenstein JM, Xu D, Zhou D, Li H, Zhang H, Li C, Qiu J and Chen X: The roles of microRNA in human cervical cancer. Arch Biochem Biophys 690: 108480, 2020.

23. Cao D, Wang Y, Li D and Wang L: Reconstruction and analysis of the differentially expressed IncRNA-miRNA-mRNA Network based on competitive endogenous RNA in hepatocellular carcinoma. Crit Rev Eukaryot Gene Expr 29: 539-549, 2019.

24. Sun Z, Jing C, Xiao C and Li T: An autophagy-related long non-coding RNA prognostic signature accurately predicts survival outcomes in bladder urothelial carcinoma patients. Aging 12: 15624-15637, 2020.

25. Saleh M, Virarkar M, Javadi S, Elsherif SB, de Castro Faria S and Bhosale P: Cervical cancer: 2018 revised international federation of gynecology and obstetrics staging system and the role of imaging. AJR Am J Roentgenol 214: 1182-1195, 2020

26. Cancer Genome Atlas Research Network; Albert Einstein College of Medicine; Analytical Biological Services; Barretos Cancer Hospital; Baylor College of Medicine; Beckman Research Institute of City of Hope; Buck Institute for Research on Aging; Canada's Michael Smith Genome Sciences Centre; Harvard Medical School; Helen F. Graham Cancer Center \& Research Institute at Christiana Care Health Services, et al: Integrated genomic and molecular characterization of cervical cancer. Nature 543: 378-384, 2017.

27. Livak KJ and Schmittgen TD: Analysis of relative gene expression data using real-time quantitative PCR and the 2(-Delta Delta C(T)) method. Methods 25: 402-408, 2001.

28. Zhang Y, Wang D, Wu D, Zhang D and Sun M: Long noncoding RNA KCNMB2-AS1 stabilized by $\mathrm{N}^{6}$-methyladenosine modification promotes cervical cancer growth through acting as a competing endogenous RNA. Cell Transplant 29: 963689720964382,2020

29. Min $\mathrm{H}$ and $\mathrm{He} \mathrm{W}$ : Long non-coding RNA ARAP1-AS1 promotes the proliferation and migration in cervical cancer through epigenetic regulation of DUSP5. Cancer Biol Ther 21: 907-914, 2020 .

30. Wang AH, Zhao JM, Du J, Pang QX and Wang MQ: Long noncoding RNA LUCAT1 promotes cervical cancer cell proliferation and invasion by upregulating MTA1. Eur Rev Med Pharmacol Sci 24: 8623, 2020
31. Taheri $\mathrm{M}$ and Ghafouri-Fard S: Long Non-cod-ing RNA signature in cervical cancer. Klin Onkol 31: 403-408, 2018.

32. Bu D, Yu K, Sun S, Xie C, Skogerb $\varnothing$ G, Miao R, Xiao H, Liao Q, Luo H, Zhao G, et al: NONCODE v3.0: Integrative annotation of long noncoding RNAs. Nucleic Acids Res 40: D210-D215, 2012.

33. Braga EA, Fridman MV, Moscovtsev AA, Filippova EA, Dmitriev AA and Kushlinskii NE: LncRNAs in ovarian cancer progression, metastasis, and main pathways: ceRNA and alternative mechanisms. Int J Mol Sci 21: 8855, 2020.

34. Zheng D, Dai Y, Wang S and Xing X: MicroRNA-299-3p promotes the sensibility of lung cancer to doxorubicin through directly targeting ABCE1. Int J Clin Exp Pathol 8: 10072-10081, 2015.

35. Wang JY, Jiang JB, Li Y, Wang YL and Dai Y: MicroRNA-299-3p suppresses proliferation and invasion by targeting VEGFA in human colon carcinoma. Biomed Pharmacother 93: 1047-1054, 2017.

36. Zhao R, Liu Q and Lou C: MicroRNA-299-3p regulates proliferation, migration and invasion of human ovarian cancer cells by modulating the expression of OCT4. Arch Biochem Biophys 651: 21-27, 2018

37. Yu Y, Zhao JD and Yang H: MiR-299-3p inhibits proliferation and invasion of cervical cancer cell via targeting TCF4. Eur Rev Med Pharmacol Sci 23: 5621-5627, 2019.

38. Li X, Ma N, Zhang Y, Wei H, Zhang H, Pang X, Li X, Wu D, Wang D, Yang Z and Zhang S: Circular RNA circNRIP1 promotes migration and invasion in cervical cancer by sponging miR-629-3p and regulating the PTP4A1/ERK1/2 pathway. Cell Death Dis 11: 399, 2020.

39. Dong J, Sui L, Wang Q, Chen M and Sun H: MicroRNA-26a inhibits cell proliferation and invasion of cervical cancer cells by targeting protein tyrosine phosphatase type IVA 1 . Mol Med Rep 10: 1426-1432, 2014

40. Li C, Jiang Y, Miao R, Qu K, Zhang J and Liu C: MicroRNA-1271 functions as a metastasis and epithelial-mesenchymal transition inhibitor in human HCC by targeting the PTP4A1/c-Src axis. Int J Oncol 52: 536-546, 2018.

This work is licensed under a Creative Commons Attribution-NonCommercial-NoDerivatives 4.0 International (CC BY-NC-ND 4.0) License. 